\title{
Identification du gène du syndrome de Purtilo
}

Le syndrome de lymphoprolifération liée à l'X (LPX), décrit par D. Purtilo il y a maintenant près de 30 ans, est lié à une infection chronique par le virus d'Epstein-Barr (EBV) chez des sujets ayant un déficit héréditaire de la réponse immunitaire spécifique vis-à-vis de ce virus. Les manifestations apparaissent dans l'enfance, chez des sujets masculins, et sont liés à une prolifération non contrôlée de lymphocytes T et B liée à l'infection par l'EBV. Près de la moitié des patients décèdent dans un tableau clinique rappelant la mononucléose infectieuse, un tiers des patients développent une hypogammaglobulinémie au décours de l'infection par l'EBV, et une forte proportion des patients développent un lymphome malin lié à l'EBV. La majorité des patients décèdent avant l'âge de 40 ans. Par une approche génétique, on a localisé le locus du syndrome du Purtilo dans la région Xq24-25, mais toutes les tentatives pour cloner le gène ont été infructueuses jusqu'à présent.

Tant sur le plan clinique qu'anatomopathologique, les manifestations du syndrome de LPX rappellent les proliférations malignes de l'EBV, rencontrées chez les patients immunodéprimés chroniques après transplantation d'organe ou greffe de moelle. Cela illustre le rôle indispensable du système immunitaire dans le contrôle de l'infection par l'EBV. Après la primo-infection qui survient, de manière symptomatique ou non, chez la quasi-totalité des sujets, s'installe une relation hôte-virus largement contrôlée par les effecteurs lymphocytaires $\mathrm{T}$ spécifiques du virus. Cela est démontré de manière éclatante par la guérison d'authen- tiques proliférations tumorales de lymphocytes B liées à l'EBV survenant après greffe de moelle chez les sujets immunodéprimés, lors de la réinjection de lymphocytes ou l'arrêt des médicaments immunosuppresseurs.

\section{Caractérisation du gène du syndrome de LPX}

Deux articles récents [1, 2] font état de la caractérisation et du rôle fonctionnel du gène du syndrome de LPX. Le clonage positionnel du gène a été réalisé par une large coopération européenne [1]. Ce gène possède 4 exons et code pour une protéine de 120 acides aminés contenant un domaine SH2 (Src homology-2). Le gène dénommé $S H 2 D 1 A$ est exprimé dans le thymus et le poumon et par les lymphocytes. Une mutation de ce gène a été trouvée dans 9 cas de LPX sur 16 étudiés.

Dans un travail très élégant, Sayos $e t$ al. (Boston, MA, USA) [2] ont également caractérisé le gène du syndrome de Purtilo mais par une approche totalement différente. En effet, cette équipe s'est initialement intéressée à la protéine CDw150, appelée SLAM (signalling lymphocyteactivation molecule) qui est une molécule transmembranaire présente à la surface des lymphocytes T et B. SLAM est son propre ligand et joue un rôle important dans la stimulation bidirectionnelle lors du contact entre les lymphocytes $\mathrm{T}$ et $\mathrm{B}$. La stimulation de SLAM induit la prolifération des lymphocytes T humains mémoires (CD45 $\mathrm{RO})$, potentialise la stimulation des lymphocytes $\mathrm{T}$ par le récepteur $\mathrm{T}$ ou par les complexes CMH-peptides, induit la synthèse de cytokines de type 1 comme l'interféron $\gamma$ et est capable de diriger une réponse de type TH2 vers un phénotype TH1 ou TH0. La stimulation des lymphocytes B par la forme soluble de SLAM, ou la forme exprimée par des lymphocytes transfectés, augmente de façon considérable la prolifération des lymphocytes B et la production d'anticorps en association avec une stimulation par la voie du CD40. Enfin, l'interaction SLAM-SLAM lors des interactions des lymphocytes B et $\mathrm{T}$ induit la prolifération et la différenciation de lymphocytes $B$ activés. Pour déterminer par quel mécanisme SLAM induit un signal de prolifération, Sayos et al. ont utilisé un système de double hybride contenant la partie cytoplasmique de la molécule SLAM et une bibliothèque d'ADNc de lymphocytes $\mathrm{T}$, à la recherche de protéines susceptibles d'interagir avec SLAM. Cela leur a permis d'isoler 8 clones codant pour une protéine de 128 acides aminés qu'ils ont nommée SAP (SLAM-associated protein). Cette toute petite protéine possède un domaine SH2 suivi d'une queue de petite taille (26acides aminés) qui n'a pas d'analogue dans les banques de données. SAP est synthétisée par les lymphocytes $\mathrm{T}$, mais non par les lymphocytes B, chez la souris et l'homme. L'utilisation d'un clone contenant les 4 exons de la SAP murine a permis à cette équipe de localiser le gène au niveau de la région A51 du chromosome X chez la souris correspondant au locus Xq25 chez l'homme, c'est-à-dire dans la même région que le gène impliqué dans le syndrome LPX.

La confirmation que SAP est codée par le gène impliqué dans le syndrome LPX a été obtenue par l'étude 
des lymphocytes de trois patients atteints de syndrome de Purtilo. Dans les trois cas, les ADNc étudiés étaient mutés, ou délétés au niveau du $2^{\mathrm{e}}$ ou $\mathrm{du} 3^{\mathrm{e}}$ exon du gène. Ce type d'anomalie n'a jamais été observé chez les sujets normaux ou les membres masculins de la fratrie non malades, ce qui confirme que les mutations mises en évidence au niveau du gène de SAP ne correspondent pas à un polymorphisme génétique.

\section{Une interprétation du syndrome LPX}

Par une approche fonctionnelle, Sayos et al. ont montré que SLAM et SAP interagissent par leur domaine SH2. En fait, la protéine SAP bloque l'interaction de SLAM avec la tyrosine phosphatase SHP2. Or cette interaction, qui permet le recrutement à la membrane de la phosphatase SHP2, interrompt la transmission du signal. La liaison SAP-SLAM a donc un effet positif sur la cascade de signalisation. La stimulation par des anticorps anti-SLAM et anti-CD3 de cellules Jurkatt transfectées par SLAM et exprimant SAP augmente significativement l'activation de ces cellules. Les auteurs suggèrent donc que les mutations de SAP observées chez les patients ayant un syndrome de PLX, interfèrent avec la transmission du signal déclenché lors des interactions relayées par les molécules SLAM à l'interface des cellules T et B infectées par l'EBV. En effet, les lymphocytes B infectés par l'EBV expriment fortement CDw150 et sont les cellules présentatrices de l'antigène aux lymphocytes $\mathrm{T} \mathrm{CD} 4$ et $\mathrm{CD} 8$ spécifiques des protéines de l'EBV. L'absence d'interaction entre SLAM et SAP gênerait la mise en ouvre des réponses $\mathrm{T}$ spécifiques nécessaires à l'élimination des lymphocytes B infectés par l'EBV. Cela pourrait expliquer certaines des anomalies immunologiques mises en évidence dans le syndrome de Purtilo, comme la diminution de la production d'interféron $\gamma$ par les lymphocytes $T$ CD4 et de lymphocytes T cytotoxiques spécifiques de l'EBV.

Ces résultats expliquent de manière incomplète l'observation curieuse de la persistance, chez les patients ayant un syndrome de Purtilo, de réponses lymphocytaires $\mathrm{T}$ spécifiques en présence d'autres virus que l'EBV. Un certain nombre de questions restent en suspens, comme l'existence éventuelle d'une régulation différente du circuit d'activation SLAM-SAP en fonction de l'âge ou du type de réponse immunitaire pouvant expli- quer l'existence d'une infection EBV symptomatique comme la mononucléose infectieuse chez les adolescents, ou totalement asymptomatique à d'autres moments de la vie. Enfin, comme le soulignent les auteurs, il peut être intéressant d'étudier la régulation de cette voie d'activation particulière du lymphocyte $T$ lors de maladies liées à l'EBV comme les proliférations polyclonales ou lymphomateuses observées chez les patients ayant un déficit immunitaire (SIDA) ou secondaires à l'utilisation de traitements immunosuppresseurs (greffe de moelle, transplantation d'organe). On pourrait imaginer que dans ces situations "l'effet transformant » de l'EBV puisse s'exprimer en raison d'une anomalie de la régulation du gène $S A P$ ou du circuit d'activation CDw150-SAP.

Y.L.

1. Coffey AJ, Brooksbank RA, Brandau O, et al. Host response to EBV infection in X-linked lymphoproliferative disease results from mutations in an SH2-domain encoding gene. Nat Genet 1998; $20: 129-35$.

2. Sayos J, Wu C, Morra M, et al. The X-linked lymphoproliferative-disease gene product SAP regulates signals induced through the co-receptor SLAM. Nature 1998: 395: 462-9.

\section{Sixième NAT (Nant s/Actualités/Transplantation) 10-11 juin 1999 - NANTES (France) Targeting Recipient Immune Response through Bioreagents}
B. Malissen
- Molecular interaction in first signal.
T. Strom
- Manipulation of allo-recognition through T-cell growth factor.
J. Thomas
- Tolerance induction using anti-CD3 immunotoxine antibodies.
L. Chatenoud
R. Zhong
J. Bluestone
- Immuno-intervention through lymphocyte receptor targeting.
D. Latinne
T.C. Pearson
S. Knechtle
A. Wörn
B. Vanhove
R. Dunbar
G. Grassy
N. Suciu-Foca
F. Sanfilippo
- Prevention rejection and induction of tolerance by monoclonal antibodies against CD45RB.
- Second signals in allo and xeno-recognition.
- Immuno-intervention through CD2/LFA3 inhibition.
- Manipulation of B7/CD28-CTL44 in primates.
- Inhibition of CD40 L pathway.
- Engineering of scFv fragments for extracellular and intracellular applications.
- ScFv and intra-cellular bioreagents.
- HLA tetramers.
- Computer-assisted rational design of immunosuppressive peptides.
J.S. Pober
- Altered Peptides in vivo.
- Inhibition of complement in vivo.
- Targeting second signals provided by vascular endothelial cells.

Renseignements et formulaires d'Abstracts : NAT Secrétariat ITERT, CHU Hôtel-Dieu, 30, boulevard Jean-Monnet, 44093 Nantes (France). Fax: (33) 2400874 11. Inscriptions: 1900FF (déjeuners et dîners compris).

Date limite de remise des abstracts: $1^{\text {er }}$ avril 1999. 